\title{
El currículum de las costumbres dóciles o de cómo Chile eliminó la ignorancia y aprendió a contener el desacuerdo en los estrictos bordes del mercado como verdad'
}

\section{The curricula of obedient habits or how Chile removed ignorance and learned to contain the disagreement in the strict borders of market as a truth}

\section{0 curriculum dos costumes dóceis o de como o Chile eli- minou a ignorância e aprendeu a conter o desacordo nas estritas margens do mercado como verdade}

Mario Sobarzo M. ${ }^{2}$

Recibido: 31 de agosto de 2018 . Aceptado: 8 de octubre de 2017

\begin{abstract}
Resumen
En este artículo se aborda la problemática que genera la comprensión del conflicto educativo chileno, el más persistente en la historia nacional a pesar de que sus actores y las ideas que los motivaron han cambiado radicalmente en el tiempo. De este modo, se intenta comprender la incógnita acerca de por qué este conflicto ha tenido tanta relevancia, a pesar de que los estándares internacionales muestran al sistema educativo chileno como uno de los mejores a nivel latinoamericano. Se busca interpretar la contradicción entre un sistema que ha tendido a transformar la sociedad chilena en un sistema de castas, pero que a pesar de ello establece vínculos cívicos poco conflictivos, lo que es extraño en los sistemas sociales de este tipo, según Alexis de Tocqueville.
\end{abstract}

Palabras clave: Costumbres dóciles - Conflicto educativo en Chile Desigualdad social y educación - Desacuerdo

1 Este artículo fue presentado en la Jornada de estudios internacional Jacques Rancière. Repartos de lo sensible, organizado por la Universidad de Valparaíso, el 10 de noviembre del año 2016.

2 Chileno, Doctor en Filosofía Moral y Política de la Universidad de Chile. Académico de la Universidad de Santiago de Chile (USACH). Contacto: mario.sobarzo.m@usach.cl 


\begin{abstract}
This article addresses issues generated by understanding the Chilean education conflict, the most persistent in national history, despite its actors and motivations have changed radically over time. In this way, we try to understand the question about why this conflict has been so relevant, despite international standards show Chilean educational system as one of the best in Latin America. It seeks to interpret the contradictions in a system tending to transform Chilean society into a caste system, nevertheless establishing little conflictive civic links which, according to Alexis de Tocqueville, is something particular in this type of social systems.
\end{abstract}

Keywords: Obedient habits - Education conflict in Chile - Social inequity and education - Disagreement

\title{
Resumo
}

Neste artigo aborda-se a problemática que gera a compreensão do conflito educativo chileno, o mais persistente na história nacional, apesar de que os seus atores e as ideias que os motivaram têm mudado radicalmente no tempo. Deste modo, tenta-se compreender a incógnita acerca de por que este conflito tem tido tanta relevância, apesar de que os critérios internacionais mostram o sistema educativo chileno como um dos melhores a nível latino-americano. Busca-se interpretar a contradição entre um sistema que tem tendido a transformar a sociedade chilena em um sistema de castas, mas que apesar daquilo estabelece vínculos cívicos pouco problemáticos, o que é estranho nos sistemas sociais deste tipo, segundo Alexis de Tocqueville.

Palavras-chave: Costumes dóceis - Conflito educativo no Chile - Desigualdade social e educação - Desacordo

Así, la democracia no solamente hace olvidar a cada hombre a sus abuelos; además, le oculta sus descendientes y lo separa de sus con-

temporáneos. Lo conduce sin cesar hacia sí mismo y amenaza con encerrarlo en la soledad de su propio corazón.

Alexis de Tocqueville

\section{Las anomalías del conflicto por la educación en Chile}

Cuando se observa la sociedad chilena y su sistema educativo, llama la atención la distancia existente entre los conflictos latentes y explícitos, versus la capacidad de desarrollar acuerdos por parte de los grupos 
con poder económico y político, en relación a los objetivos que se le plantean a los distintos ámbitos que estructuran la modalidad de enseñanza implementada y legitimada.

Desde 2001 en adelante la educación chilena ha vuelto a tener protagonismo como escenario de conflicto. La principal terminología asumida por los distintos actores con capacidad legislativa ha estado concentrada y estructurada en relación con el problema de la inclusión, la igualdad de oportunidades y el desarrollo de una calidad semejante para los distintos actores que integran las modalidades educativas existentes. Programas como el de Acompañamiento y Acceso Efectivo a la Educación Superior, PACE, o la incorporación del ranking en educación superior, o la ampliación de la subvención como modo de reemplazar el Financiamiento Compartido, FICOM, dan cuenta de que esta forma de operar es transversal y representa una política macro, que incluso tiene alcances internacionales (Verger). Esta línea de análisis e implementación de políticas ha permitido el desarrollo de un modelo educativo que logra insertarse en Latinoamérica como uno de los mejores en enseñanza universitaria, secundaria e incluso primaria y preescolar. Así, desde el punto de vista de los estándares internacionales, la educación chilena descolla frente a la de sus vecinos latinoamericanos, aunque queda rezagada respecto del resto de los integrantes de la Organización para la Cooperación y el Desarrollo Económico, $\mathrm{OCDE}^{3}$. Es por ello que, internacionalmente, llama la atención la continua conflictividad social que año a año se desarrolla

3 Respecto del sistema universitario, se puede citar el ranking de la consultora inglesa Quacquarelli Symonds (QS) que ubica a 11 universidades nacionales, en 2017, entre las 500 mejores del mundo, y a dos entre las 10 mejores de Latinoamérica. También el elaborado por la revista Times Higher Education (THE), que sitúa a Chile como uno de los cuatro países líderes en la región, después de Brasil. En el ranking de Shangai, recientemente publicado, tres universidades chilenas aparecen como líderes a nivel latinoamericano, situándose por encima de las brasileñas en varias áreas de estudio. Desde el punto de vista escolar, la prueba PISA del año 2015 muestra que Chile tiene mejores desempeños que el resto de los países latinoamericanos evaluados, superando a México, Perú, Colombia, Costa Rica, Brasil, República Dominicana y Uruguay. Argentina sólo evaluó a la ciudad de Buenos Aires, la que cuenta con el mayor índice de desarrollo humano, por lo que no es referencial. 
en torno al sistema educativo existente. ¿Cómo se entiende que un sistema con muy buen desempeño regional tenga tanta resistencia social, cuando no sucede lo mismo en otros países de Latinoamérica? ¿Qué articulaciones culturales y sociales se expresan detrás de las contradicciones que la educación manifiesta como espacio de conflictividad social? ¿Quiénes son los sujetos que emergen detrás de las acciones políticas y que impugnan la estructuración social que se expresa en el sistema educativo? Y, en último término, ¿por qué estas contradicciones sociales tienen su espacio privilegiado de expresión en el ámbito educativo?

Cuando se analiza la historia del sistema educativo chileno, es posible observar semejanzas con la evolución que tuvieron las luchas sociales por la educación en Latinoamérica. Desde los conflictos entre conservadores y liberales, en los inicios de las repúblicas, hasta la importancia de la universidad como espacio privilegiado para la construcción de proyectos desarrollistas nacionales, en las décadas de los 60 y 70; luego, el protagonismo que tuvieron las y los estudiantes universitarios y secundarios en la lucha contra la dictadura, hasta las masivas marchas del año 2011, la educación ha sido un hilo articulador de las demandas y proyectos políticos con capacidad de hegemonía ideológica y social. La lucha por la educación ha sido una constante en la sociedad chilena desde que se constituyera como República en el siglo XIX. Mientras en el resto de Latinoamérica los conflictos han sido espaciados y motivados por circunstancias específicas ${ }^{4}$, en Chile han estado en el centro de todos los enfrentamientos entre los proyectos modernizadores y las fuerzas conservadoras que desean mantener el statu quo social.

Mientras en países como Brasil o México el tema más relevante para la conflictividad social es el acceso a la tierra y la lucha contra la hacienda

4 Por ejemplo, las movilizaciones de las y los estudiantes argentinos durante el gobierno de Carlos Menem, cuando intentó establecer un arancel en las universidades estatales. O las movilizaciones en Colombia en 2011 por motivos semejantes a los de Argentina. 
colonial5 $^{5}$; o en lugares como Argentina, con un alto nivel de proletariado urbano y ligado a la industria, las luchas se han concentrado en los derechos laborales y la capacidad de organización del movimiento de trabajadores; en Chile la hebra que une las distintas épocas se ha concentrado en la lucha por la educación. Por supuesto, esto no quiere decir que la educación no sea, o no haya sido, un espacio de conflicto en otros países y sociedades; la importancia del "grito de Córdoba", en 1918, en Argentina trascendió a nivel latinoamericano. La politización de las universidades después de la revolución cubana o las luchas por la alfabetización campesina en Brasil no sólo fueron eventos nacionales, sino latinoamericanos, y Chile no estuvo ajeno a ellos. Sin embargo, la principal diferencia en todas estas situaciones es que la educación fue más el campo de batalla que el objetivo estratégico que motivó el conflicto.

La alfabetización campesina en Brasil era parte del desarrollo de la reforma agraria. El "grito de Córdoba" es comprensible en una sociedad en rápida expansión demográfica, ligada a la inmigración y las ideas socialistas, comunistas y anarquistas venidas con los obreros europeos que llegaron a integrarse en el emergente sistema industrial argentino. Es decir, más que objetivos en sí mismos, las necesidades de transformación del sistema educativo obedecían, en estas luchas, a campos de batalla por la cultura, como un modo de transformación ideológico y político de las sociedades, siguiendo la tesis de que el sistema educativo es el principal reproductor o formador de los constructos e ideas que articulan los vínculos y encuentros al interior de las sociedades.

Pero, la situación de Chile es un tanto distinta. Aunque efectivamente las escuelas consolidadas o las luchas de los docentes a inicios del siglo XX, o la reforma universitaria, no fueron conflictos en sí mismos, sino que estuvieron vinculados a luchas sociales más amplias, lo que resulta distinto es la persistencia en el tiempo del conflicto social por la educación, a pesar de las estadísticas internacionales que citába-

5 Piénsese en el Movimiento Zapatista o en las tomas de terreno, hasta el día de hoy, por parte del Movimiento Sin Tierra en Brasil. 
mos previamente. ¿Por qué la sociedad chilena no está satisfecha con sus logros educativos? Y, principalmente, ¿por qué la educación se constituye en el lugar de conflicto más visible, atravesando la historia nacional desde los inicios de la República?

La tesis central de este texto se basa en este problema. Considero que para entender la persistencia del conflicto respecto de la educación, al lado de otros que fueron relevantes durante el siglo XX (como el conflicto por la obtención de derechos mínimos en el trabajo, el acceso a la vivienda y la lucha por la ciudad) pero que fueron quedando desplazados y perdiendo fuerza movilizadora después de la recuperación de la democracia, hay que situar las luchas en torno a ella considerando tres factores que están concatenados. En primer lugar, la consideración de los conflictos por la educación como expresión de un desacuerdo y no sólo como espacio para la lucha social. En segundo lugar, la contradicción entre la promesa ideológica instalada por la burguesía desarrollista y con tintes nacionalistas en los siglos XIX y XX de una educación integradora y modernizante, versus la construcción y defensa ideológica de una educación diferenciada por castas, de parte de la nueva burguesía emergida después del proceso de acumulación originaria de la dictadura de Pinochet. En tercer lugar, el rol de articulación subjetiva que produce la conflictividad educativa, y especialmente, la del movimiento secundario.

En lo que sigue abordaré las problemáticas expuestas interpretadas a partir del aporte del pensamiento de Jacques Rancière, debido a que sus conceptos y desarrollos reflexivos permiten entender las contradicciones y situarlas en el marco de un conflicto político, es decir, por el reconocimiento en tanto sujeto.

\section{Sistemas de castas y costumbres}

El año 1831 el aristócrata francés Alexis de Tocqueville viaja a EEUU para investigar su sistema penitenciario. Durante su estadía en este país escribe una obra central de la filosofía política: La democracia en América (Tocqueville 2001). En ese libro se va entretejiendo un relato acerca de por qué la democracia podía existir como un sistema po- 
lítico tan exitoso en los Estados Unidos. Luego de su análisis sobre el sistema institucional, las funciones y regulaciones del poder estatal, el autor pasa a analizar un fenómeno que considera incluso más relevante que las leyes: las costumbres. En esto se entronca con la larga tradición que considera que a la política la define el modo de ser de las personas, más que el ámbito institucional ${ }^{6}$.

Casi 160 años después Jacques Rancière retoma una figura que el autor de La democracia en América había considerado fundamental: las costumbres dóciles o suaves -dependiendo de la traducción- que expresan una tendencia a la normalización de la igualdad, lo que permite limitar la ferocidad en las relaciones sociales, que primaría en los sistemas de casta vinculados al honor, y a la desigualdad.

En este texto pretendo vincular el mismo concepto, mediado por los 160 años, pero, más aún, mediado por la actualidad y contingencia que señalé en la presentación.

El concepto de "costumbre dócil" (o suave) es desarrollado por Tocqueville al inicio de la tercera parte del segundo volumen de la obra que hemos citado. Sin embargo, ya en el primer volumen desarrolla ampliamente la importancia que tienen las costumbres, llegando en el segundo a afirmar que el ámbito de lo físico, relacionado con lo legal y esto con las costumbres (en el caso de Norteamérica), influye directamente en el espíritu democrático, es decir igualitario. ¿A qué se debe esta tendencia? ¿A la morigeración o a la docilidad?

\footnotetext{
6 Sólo por citar algunas obras y autores relevantes acerca de este tema, comenzando por Platón, quien señala la importancia de las formas de vida en la educación de los ciudadanos de la República ideal, tanto en Las leyes como en el libro homónimo, La República. También Aristóteles enfatiza la importancia de la educación y las costumbres en su Política y en la Ética a Nicómaco, donde incluso señala el vínculo entre la palabra "costumbre", escrita con épsilon, y la palabra "carácter", escrita con eta, lo que implicaría una relación más profunda que la mera homofonía de ambas, vinculando su enseñanza con la expresión cívica y activa de ellas. La tradición de este vínculo es retomada posteriormente por Cicerón, los estoicos romanos, Maquiavelo y Montesquieu, entre muchos otros.
} 


\begin{abstract}
"Las instituciones feudales hacían muy sensibles los males de ciertos hombres, pero no las miserias de la especie humana. Prestaban más bien generosidad que dulzura a las costumbres, y aunque surgiesen grandes sacrificios, no hacían nacer verdaderas simpatías; pues no hay simpatías reales sino entre personas semejantes, y en los siglos aristocráticos no se consideran como tales sino los miembros de la misma casta.
\end{abstract}

Cuando los cronistas de la edad media, que pertenecían todos por su nacimiento o por sus hábitos a la aristocracia, refieren el fin trágico de un noble, lo hacen con mucho dolor; más nos cuentan la matanza y los tormentos de la gente del pueblo sin emoción y sin relieve alguno." (Tocqueville 2001 520).

Luego de este análisis sociológico y filosófico-político, y para dejar completamente claro lo que está señalando con esta idea, Tocqueville introduce la cita a una carta escrita en 1675 por una mujer noble, Madame de Sévigné. La carta contrasta por la afectividad que presenta hacia su hija (la persona hacia quien va dirigida), versus la descripción de los horrores cometidos contra una rebelión de la Bretaña. Esta última descripción tiene casi una rigurosidad científica, para dar paso luego a una nimiedad respecto de otra señora que visitó los bosques.

"Anteayer murió en el suplicio de la rueda el que tocaba el violín cuando empezó la bulla y el robo del papel sellado, lo descuartizaron y expusieron sus miembros en los cuatro ángulos de la ciudad. Han arrestado a sesenta vecinos y desde mañana empezarán a ahorcarlos. Esta provincia es un lindo ejemplo para las otras, y principalmente para enseñar a respetar a los gobernadores y gobernadoras, y a no tirar piedras a su tejado.

Madame de Tarente estuvo ayer en estos bosques con un tiempo delicioso; pero no hay que hablarle de habitación ni de comida, pues entra por una puerta y vuelve a salir por la otra (...)" (Tocqueville 2001 520-521). 
Nuestro autor no pretende juzgar la dureza de las palabras utilizadas por Madame de Sévigné, sino, inversamente, intenta mostrar que la persona que las refiere no tiene un vínculo humanitario con los actos que se cometen contra los miserables que hicieron la rebelión. Esto no es culpa de ella, sino del lugar y las personas con las que se vincula. Por ello Tocqueville señala que, incluso si la crueldad y la burla contra el sufrimiento ajeno pudiera ser tolerada (en la época en que escribe su libro) por parte de un grupo social, la tendencia igualitaria de la sociedad en general le impediría realizar este tipo de actos.

En palabras de nuestro autor, la tendencia a la igualdad genera vínculos más empáticos, pues tendemos a concebir a los otros como iguales a nosotros, sin importar su nacimiento o condición. Más aún en sociedades en que las condiciones de igualdad están garantizadas por la prosperidad económica, como es el caso de la norteamericana a fines del siglo XVII e inicios de XVIII. Sin embargo, no se preste a engaños: como lo ha señalado en el primer volumen, la igualdad excesiva tiende a llevar al "individualismo". Para el autor, este concepto no puede ser confundido con el de "egoísmo" pues, aunque comparten un elemento común, que es la relación del individuo consigo mismo, uno tiende a ser una separación producto del miedo hacia el otro, la competencia con él, mientras que el segundo (individualismo), nace de la despreocupación, la desafección con el otro, la indiferencia.

Un primer aspecto que se podría señalar es justamente este punto de separación, de partición, que representa el límite entre la suavidad o docilidad con los demás y la relación con la indiferencia del individualismo. Más aún si consideramos que el cierre del capítulo citado termina situando el punto de exclusión respecto de este principio de semejanza que genera la igualdad: la esclavitud.

Insisto en esto: ¿mediante qué proceso una sociedad igualitaria como la norteamericana, según Tocqueville, donde casi todas las clases son iguales y por ende los hombres tienden a pensar y sentir de modo semejante, donde las desdichas ajenas generan un instinto secreto de piedad, sin importar si el otro es extranjero o enemigo, no sólo tolera, sino que incluso permite en su seno un fenómeno como la esclavitud, que si bien es, según sus palabras, la más benigna de entre todas las 
otras formas de esclavitud que existen en otros sitios del nuevo mundo, no por ello resulta menos miserable, o no implica castigos muy crueles para los "negros"? El capítulo se cierra con una comparación con estos rasgos en los pueblos, apelando a los romanos y su ferocidad para con sus enemigos, a quienes no consideraban sus iguales.

Dejaré esta descripción hasta aquí, pero la retomaré posteriormente. Ya he señalado que la idea de "costumbres dóciles" la menciona Rancière (Rancière 2011) en un libro publicado originalmente en francés en 1990, cuyo primer artículo ("El fin de la política o la utopía realista") está dedicado a una situación de contingencia: la elección presidencial de 1988. En ella el autor se interroga acerca del abandono de Mitterrand, electo ya presidente, de la promesa. Con ello no se refiere a una promesa en específico, sino a la promesa en tanto tal, la que, como señaló Arendt (2003), emana directa e indisolublemente de la libertad humana, expresada en la Acción.

De los tres candidatos analizados, no sólo Mitterrand llegó a ser presidente de Francia en dos ocasiones, sino también, posteriormente, Chirac. El tercero (Le Pen), para nuestro autor, nombrado a partir de su eslogan, era el resultado de la descomposición de la política, una doble supresión de lo político en lo social y de lo social en lo político.

"Pues lo que propone el viejo auctor es, sin duda, esa tarea de la modernidad proclamada por doquier: secularizar lo político, desmilitarizarlo, reducirlo a la simple gestión de lo social, eliminando todo aquello que no esté dirigido a la maximización de las posibilidades de éxito del ser-enconjunto.

Hacía de esta pacificación la tarea de la política. Pero, al mismo tiempo, hacía evidente la paradoja misma de la política, concebida como el arte de los gobernantes, de esa política que se había impuesto a nuestra percepción a través de la conjunción ancestral de las pequeñas prácticas gubernamentales y las grandes teorías filosóficas. La tarea de esa política se define como sustracción de lo político. Sustracción que puede ser descrita de dos maneras, según el modo en que 
se considere la relación entre las categorías de lo social y lo político. Sustraer lo político significa, en cierto sentido, reducirlo a su función pacificadora de nexo entre los individuos y la colectividad al descargarlo del peso y de los símbolos de la división social. Significa, al mismo tiempo, suprimir los símbolos de la división política en beneficio de la expansión y el dinamismo propio a la sociedad." (Rancière 2011 26- 27).

Dicha supresión tiene su punto de cesura en el discurso de la modernización, ese acuerdo que disuelve el límite entre el partido de los ricos y el partido de los pobres; la aparición de ese odio al otro, que reúne para excluir. Ese retorno de lo arcaico, que en ese artículo aún representa un temor futuro, una preocupación, un poco más de un cuarto de siglo después aparece en su materialidad más cruda en distintos escenarios electorales globales, y es posible que dentro de poco, también llegue a afectar a Chile. Es por esto que quiero detenerme en dos elementos que el artículo del profesor Rancière expone y que creo tienen plena significación en Chile.

La primera de estas ideas tiene que ver con esta borradura de lo social y lo político; la segunda, con sus implicancias en lo educativo.

Su tesis sobre este afán de borradura entre lo social y lo político, como diferencia que apela a la igualdad y a partir de ello se instituye en la palabra, en el gesto, en la acción, e incluso en la violencia y capacidad de irrupción en el espacio común, aparece como uno de los rasgos centrales para disolver lo que en su teoría política llama "el desacuerdo".

La inscripción de estos conceptos en medio de un artículo de política contingente expresa un punto de máxima cercanía entre lo que en él se señala y nuestra actualidad. Pero también un punto de ruptura, una torcedura en el espacio simbólico, en la inscripción de los cuerpos en el espacio común entre la experiencia a la cual se dirige, como análisis, el artículo (la elección presidencial de 1988 en Francia) y la actualidad del año 2016 en Chile.

Lo sabemos, ese odio al otro en Chile se expresó de modo material, pero también con simbolismos del horror que, incluso hoy, posee un 
museo. Esta situación en esos momentos en Francia recién estaba comenzando, y no había alcanzado las dimensiones que tiene en la actualidad, debido a la destrucción y recomposición política que vivieron los países árabes y subsaharianos posteriormente a lo que se ha llamado "primavera árabe". Antes de eso el horror nacionalsocialista se vivió como ocupación: era un otro que expresaba su odio y con el que era mejor no encontrarse. En Chile, en cambio, este ejercicio del horror se inició con un acto espectacular: el bombardeo de la casa de gobierno. De ahí en más, nunca la tiranía abandonó ese gusto por la espectacularidad, utilizando mecanismos como el degüello, quemar a personas, desaparecer cadáveres, entre muchas otras fórmulas que no estaban dirigidas a mantenerse en silencio, sino a irrumpir en el ámbito de la visibilidad.

Pero quiero regresar aquí a los puntos de máxima cercanía entre ambos contextos, lo que incluso por momentos tiene tonos de comedia, como cuando Rancière describe a Mitterrand como un auctor por su Carta para todos los franceses. Imposible no vincular dicho texto con el aparecido en los últimos días de Ricardo Lagos, incluso en su extensión: libro (Lagos 2017). Pero más allá de lo cómico que puede resultar que nuestro candidato intente homologar lo realizado por el presidente francés, lo interesante es la relación que se establece en el texto entre esta figura del auctor y su capacidad de apaciguar, de resolver mediante las letras el ruido de la querella, unir gente por su capacidad para discernir el sentido, pacificar en virtud de una capacidad que precede al ejercicio del poder, es alguien que permite la armonización consensual de la sociedad. En una elección siguiente, nos señala Rancière, ya ni siquiera sería necesario que el candidato escribiera una obra literaria, un texto sobre el cual coincidir, sería suficiente el miedo a la emergencia del horror, el odio al otro, para que el candidato de la modernización, sin importar que viniera de la derecha (Chirac), fuera mejor.

Interesante borde que señala un límite a nuestra actualidad. El temor a ese borde, que se pierde en algún momento, representa ese retorno a lo arcaico, un lanzarse colectivo que sólo puede existir cuando la borradura entre política y sociedad ocurre en forma perfecta, es decir, 
cuando ya nadie real puede sentirse ni cerca ni conmovido por ella. Es una desaparición del sujeto y los sujetos, que quedan reducidos a meros engranajes de una maquinaria cuyo sentido se encuentra por fuera de lo político.

Volvamos a estas dos borraduras, reducciones, que hemos citado. La primera, la que reduce lo social a lo político, trata de apaciguar mediante la distribución de derechos, cargas y controles el conflicto entre ricos y pobres; mientras que la segunda, permite el apaciguamiento de las pasiones relativas a la ocupación del centro. Respecto de esta producción ideal de lo político por lo social, se intenta vincular el centro político con la clase media, en un viejo recurso que data desde Aristóteles. El uso de la misma palabra (centro), pero sin carga política, permite la identificación con una clase social que está entre los ricos y los pobres. Es el intento de poner sutura a la división social, a la diferencia de intereses y formas de emerger en el espacio.

Esta es una forma de política autosustraída, que llega a la perfección en su capacidad de resolver los intereses individuales, la ganancia, versus los intereses públicos vinculados a la pasión por el honor, donde la máxima libertad ya no es un concepto político, sino la apolítica autosatisfacción individual que expresa una forma de mezquindad; la idiotez de los intereses privados y por ende la pérdida de la acción colectiva. Ese lugar, aunque parece una forma perfecta de democracia, ha llegado a un tipo de tiranía que ya no importa a ningún sujeto, es decir, en la que ningún colectivo es capaz de situarse simbólicamente, políticamente en acciones, emancipatoriamente en proyectos y formas de encuentro; ese lugar implica la extinción perfecta de la política. Ahí ya no hay espacio para el conflicto o el desacuerdo, pues no hay ningún punto de encuentro donde eso sea posible.

"La política es asunto de sujetos, o más bien de modos de subjetivación. Por subjetivación se entenderá la producción mediante una serie de actos de una instancia y una capacidad de enunciación que no eran identificables en un campo de experiencia dado, cuya identificación, por lo tanto, corre pareja con la nueva representación del campo 
de la experiencia (...) Un modo de subjetivación no crea sujetos ex nihilo. Los crea al transformar unas identidades definidas en el orden natural del reparto de las funciones y los lugares en instancias de experiencia de un litigio." (Rancière 1996 52).

Y es justamente el dispositivo de las costumbres dóciles de Tocqueville el que permite a nuestro autor explicar la actualidad de ese momento, descrita por algunos como posmodernidad. En ella, con su ausencia de horizonte, se expresa la realización perfecta del despotismo. Si la desaparición de la polarización entre ricos y pobres ha hecho posible la retirada de las fiebres del honor político y la democracia heroica, haciendo emerger un individualismo que vive su libertad de forma constante, en la medida en que no se topa con los demás, esto sólo puede suceder (si es que la fantasía se realiza realmente) cuando es capaz de convertirse en instrumento político para el control de lo social.

Es este el segundo aspecto que me interesa desarrollar, pues se refiere a la educación y creo que en Chile permite explicar el reflujo que vive el movimiento social hoy, justamente, con relación a ella.

El movimiento social por la educación, desarrollado entre 2001 y 2011, tuvo una capacidad de incidencia cultural, transformando los símbolos, cuestionando los modos de aparecer en el espacio y las formas de subjetividad que tenían derecho a ello, construyendo nuevos significantes y disputando el sentido de otros, reconstituyendo las formas de organización y recreando las formas de comunicación y vinculación hacia el interior de las organizaciones y entre ellas.

Sin embargo, no deja de ser relevante observar que es justamente en el ámbito educativo, en la zona de disputa acerca de lo establecido en la Constitución de Pinochet (reformada tibiamente por Lagos), que se constituye el punto de torsión ideológico, pero también la crisis del sentido que le impide ir más lejos al sujeto en su politización y la forma de subjetividad que emergió del conflicto. La fragmentación infinita de los intereses individuales, que alcanzó su punto de satisfacción en una relación directa con el servicio, un financiamiento amable y la posibilidad de disputar en un conflicto burocrático y mercantil, con 
una amplia gama de funcionarios que van desde el asistente social hasta la ejecutiva bancaria, se convierte en punto de convivencia, en zona de encuentro para la aparición de la igualdad en las formas. La zona del Barrio República, en Santiago de Chile, representa un ejemplo maravilloso de esta amalgama de individuos que se encuentra en un espacio, pero no produce ni identidad, ni singularización, ni capacidad de institución de conflicto para emerger como un sujeto. Sólo están ahí, ocupan un lugar en el espacio físico, sin llegar a su politización desde la conflictividad social.

Lo increíble de esta torsión es que expresa el fracaso de la capacidad de instituir desacuerdo por parte del okhlos, de separar y dividir el demos, rechazando la incorporación en el Uno de la colectividad. Por el contrario, es el deseo de (mediante y en la deuda) integrarse en el todo indiferenciado en que el blaberon se expresa invisiblemente, silenciosamente.

"Blaberon significa la corriente interrumpida, la torsión primera que bloquea la lógica natural de las "propiedades". Esta interrupción obliga a pensar la proporción, la analogía del cuerpo comunitario. Pero también corrompe de antemano el sueño de esta proporción." (Rancière 1996 27-28)

El movimiento social del 2006 al 2011 generó un desacuerdo, señaló que no era parte de esa totalización de las cuentas que hacía un grupo distinto, del cual el auctor era el mejor representante. Pero cinco años después la educación retornó a su condición de negocio privado, usando los mismos términos que el movimiento había transformado en su significado o creado 7 . La educación como un derecho social pasó a convertirse en un derecho individual que el Estado debe ayudar a realizar. El inicio del desacuerdo, el conflicto en las formas, controlado por el simple recurso de integrar dinero en un sistema que obliga a los jóvenes y sus familias a convertirse en esclavos de sus patrones; del sistema financiero, ante el que deben presentarse en su desnudez

7 Gratuidad universitaria, inclusión, derechos sociales, mejoramiento del sistema educativo mediante la estandarización, "control" del lucro, entre otros. 
comercial, así como del Estado, que oficia de amable garante para su ingreso en el registro de deudores; es decir, esclavos ${ }^{8}$.

\section{La clausura de lo político como corolario de la democracia chilena}

En una sociedad donde lo que impera es la "igualdad" como concepto que se integra a la escuela, que se usa para exigir derechos en la escuela (por supuesto individuales), ella sirve para enseñar la suavidad, exacerbarla por medio de los discursos (las marchas pacíficas que tanto gustan al mundo político), pero se expresa de modo radical ya no en su contradicción, sino en su aceptación como destino individual, como principio de autorrealización en la formación.

La sociedad chilena expresa esta contradicción como un espacio normalizado, como un acuerdo tácito y explícito que no queda otra posibilidad, sino aceptar. Después de todo, de esa aceptación depende la posibilidad de inclusión en un sistema donde las castas han aprendido a convivir y reunirse en torno a meras expresiones simbólicas, sin referencia, como la selección nacional masculina de fútbol, el himno devenido manifestación de identidad emotiva o los paisajes del territorio que se poseen como un sucedáneo de propiedad, que sólo es real para los capitales que son dueños hasta del agua de esos lugares. Es decir, símbolos que nos configuran experiencias sucedáneas para reemplazar y resolver el espacio de conflicto social, evitando su conversión en conflicto político.

Se evidencia así la necesidad de adecuar el tiempo cronológico de desarrollo de los niños con el tiempo mercantil. El problema es la inadecuación entre ambos, por ello todo lo que ayude a optimizar dicha relación, está en la senda correcta y las reformas implementadas por el gobierno de Bachelet como respuesta a las movilizaciones de 2011 tienden a eso. Uno de los temas centrales de la reforma llamada "gra-

8 Uno de los múltiples lienzos del 2011 decía: "la deuda es la esclavitud moderna". 
tuidad en la educación superior" fue establecer un límite de tiempo a los años en la universidad; uno de los objetivos de las primeras reformas educacionales en democracia fue bajar las tasas de repitencia. Tiempo. Cada vez que se expresa un deseo de transformación, se señala como motivo de su incapacidad de implementación el tiempo. Por supuesto que no es el tiempo de lo colectivo sino la suma de tiempos individuales, cuantificables en un contrato por deudas. Otra forma sucedánea de cuantificación del reparto desigual de lo social, del conflicto de clases, devenido expresión de una voluntad de integración o de una resistencia anómica que hay que aislar y combatir.

Sabemos lo que significa en la historia de la democracia la rebelión contra la esclavitud de la deuda, pero nunca hasta ahora habíamos cuantificado los montos de tiempos y su correlación cuantitativa con el mercado de la satisfacción. La educación y las reformas que la atraviesan en la actualidad expresan este límite difuso en el que el conflicto puede llevar a la sociedad a su conversión en sujeto y subjetividad política, pero que puede implicar también la clausura definitiva en el mero aceptar el lugar que nos tocó en el reparto de lo sensible.

Aún no logramos imaginar cuál será el resultado, pero de él depende que la hebra del conflicto social, que atraviesa la educación chilena, no se corte por varias generaciones.

\section{Bibliografía}

Arendt, Hannah. La condición humana. Buenos Aires: Paidós, 2003.

Lagos, Ricardo. Piensa en Chile. Propuestas para conversar. Santiago de Chile, 2017.

Rancière, Jacques. El desacuerdo. Política y Filosofía. Buenos Aires: Ediciones Nueva Visión, 1996.

Rancière, Jacques. En los bordes de lo político. Santiago de Chile: La Cebra, 2011.

Tocqueville, Alexis de. La democracia en América. México: Fondo de Cultura Económica, 2001. 
EL CURRÍCULUM DE LAS COSTUMBRES DÓCILES O DE CÓMO CHILE ELIMINÓ LA IGNORANCIA Y APRENDIÓ A CONTENER EL DESACUERDO EN LOS ESTRICTOS BORDES DEL MERCADO COMO VERDAD / Sobarzo

Verger, Antoni y Normand, Romuald. "Nueva gestión pública y educación: elementos teóricos y conceptuales para el estudio de un modelo de reforma educativa global". Educação y Sociedade (2015): 599-622. 